\title{
A multi-objective approach to optimize the weight and stress of the locking plates using
}

\section{finite element modeling}

\author{
Soroush Rafiei ${ }^{\mathrm{a}}$, Amir Nourani $^{\mathrm{a}^{*}}$, Mahmoud Chizari $^{\mathrm{b}}$
}

${ }^{a}$ Department of Mechanical engineering, Sharif University of Technology, Tehran, Iran

${ }^{\mathrm{b}}$ School of Engineering and Computer Sciences, University of Hertfordshire, Hatfield, UK

*Corresponding author, email address: nourani@ sharif.edu, Phone: +982166165687, Fax:

$+982166000021$

\begin{abstract}
This paper aims to identify an optimum bone fracture stabilizer. For this purpose, three design variables including the ratio of the screw diameter to the plate width at three levels, the ratio of the plate thickness to the plate width at three levels, and the diameter of the bone at two levels were selected for analysis. 18 3D verified finite element models were developed to examine the effects of these parameters on the weight, maximum displacement and maximum von Mises stress of the fixation structure. Considering the relations between the inputs and outputs using multivariate regression, a genetic algorithm was used to find the optimal choices. Results showed that the diameter of the bone and the amount of load applied on it did not have a significant effect on the normalized stresses on the structures. Furthermore, in all ratio of the plate thickness to the plate width, as the ratio of the screw diameter to the plate width increased, the amount of stress on the structure decreased. But, by further increasing the ratio of the screw diameter to the plate width, the amount of stress on the structure increased. On the other hand, by increasing the value of the ratio of the plate thickness to the plate width, the maximum amount of stress on the structure decreased. Finally, optimal solutions in terms of the weight and the maximum amount of stress on the structure were presented.
\end{abstract}


Keywords: Locking Plate; Optimization; Genetic algorithm; Finite element method; Bone fracture.

\begin{tabular}{|ll|}
\hline Nomenclatures & Diameter of the screws, $\mathrm{mm}$ \\
$d$ & Thickness of the plate, $\mathrm{mm}$ \\
$w$ & Width of the plate, $\mathrm{mm}$ \\
$v . M$ & von Mises stress, $\mathrm{MPa}$ \\
Normalized. & Normalized data, $\frac{X}{X_{\text {maximum }}}$ \\
$F_{\text {failure }}$ & Failure load, $\mathrm{N}$ \\
$F_{\text {applied }}$ & Applied force on the bone, $\mathrm{N}$ \\
$R^{2}$ & Coefficient of determination \\
\hline$\alpha$ & Constant parameter \\
$\sigma$ & Stress, MPa \\
$\sigma_{\text {ultimate }}$ & Ultimate stress, MPa \\
\hline
\end{tabular}

\section{Introduction}

Bone diaphysis fracture is a common orthopedic problem that may be caused by aging or other problems such as malnutrition, genetic disorders, traumatic injuries or accumulation of damages. ${ }^{1-}$ ${ }^{3}$ Generally, in young people (i.e. under the age of 30), this fracture is more likely to occur as a result of severe accidents, while in elderlies it would mostly happen because of falls. ${ }^{4}$ Using locking compression plates (LCPs) is a common method to stabilize internal bone fractures. These plates would allow for early ossification, whereas in the neutral plates, first a callus forms and then the ossification occurs. Therefore, they have a great advantage over other plates. ${ }^{5-7}$ However, an important challenge with this type of plates is the risk of fixation failures as widely reported by many investigators. Some studies have been done about this risk and the failure loads of these plates under various conditions have been estimated. For example, ref. ${ }^{8}$ investigated the effect of a gap between the LCP and bone on the failure load of the fixation structure. The results showed that with increasing the gap from 0 to $5 \mathrm{~mm}$, the failure load decreased by about $62 \%$. Ref. ${ }^{9}$ 
statistically examined an LCP that were used as a fixation for distal femur fractures. The results showed that $12.5 \%$ of the total fractures occurred in the first 6 weeks and $50 \%$ of them occurred after 6 months. According to this report, analyzing the strength of implants before surgery can be very helpful as the failure of the plates after surgery would cause serious problems for the patient.

Multi-objective optimization has been widely used as a way to achieve the optimum solutions for designing and improving performance of various structures; e.g. designing thin-walled structures under compressive loads. ${ }^{10,11}$ Ref. ${ }^{12}$ investigated the optimal location and number of screws to reduce the maximum displacement of LCP structures by using particle swarm optimization (PSO) algorithm. The results showed that by increasing the number of screws up to 6, with 3 screws at the top of the fracture side and 3 screws at the bottom side, the maximum displacement of structure significantly reduced. However, further growth in the number of screws had no significant effect on the maximum displacement. Mouzen et al. ${ }^{13}$ observed that configuration of screws had a greater effect on the quality of fixation than did the plate material. Wee ${ }^{14}$ simulated the plate-screw fixation system so that the bone was modeled with a homogeneous cylinder; the results showed that the distance between the screws had a significant effect on the stresses of the plate and screws.

Considering the outcomes of the studies mentioned above, since post-surgery failures of the plate is probable, it has been suggested to establish a finite element (FE) analysis before an operation to prevent unexpected implant failures. ${ }^{15} \mathrm{FE}$ analysis and other numerical simulations have been widely used as a reliable and low-cost method for recognizing and studying various phenomena such as impact and crushing problems. ${ }^{16-19}$

Recently, with the benefit of 3D printers, many implants made of titanium, ceramic etc. can be customized according to the patient bones anatomy/geometry. ${ }^{20}$ Therefore, key design parameters and their influences on the quality of fixation should be investigated. 
Up to now, evaluating the simultaneous effects of the ratio of screw diameter to plate width, ratio of plate thickness to plate width and bone diameter on the stress distribution have not been investigated. Therefore, the main objective of this study is to propose non-dominated solutions in terms of structure weight and maximum von Mises (v.M) stress using a multi objective optimization algorithm. The optimization algorithm utilized in the study is commonly used in many fields of research due to its efficiency and ability to incorporate a large number of variables. The method generally consists of four stages of initialization, crossover, selection and mutation. ${ }^{21}$ The framework presented in this study can be applied to other types of plates as well.

\section{Materials and Methods}

SolidWorks (Dassault Systèmes) software was used to model the bones, screws and plates. The threads of the screws were omitted following the approach of refs. ${ }^{22,23,24}$ and they were modeled with a diameter changing from 2 to $15 \mathrm{~mm}$ and a length of 32 to $70 \mathrm{~mm}$, according to the thickness of the plate and the diameter of the bone. The bones were modeled as a hollow cylinder 14, 24,25 with a thickness of $1.5 \mathrm{~mm}$, and a $10 \mathrm{~mm}$ transverse gap from the fracture edge was assumed in the models. ${ }^{8}$ Figure. 1 shows a schematic of the plate. The width of the plate was chosen so that the ratio of the screws diameter to plate width $(\mathrm{d} / \mathrm{w})$ was equal to $11 \%, 46 \%$ and $86 \%$ for screw diameters of 2, 8 and $15 \mathrm{~mm}$, respectively. Also, the ratios of the plate thickness to the plate width (t/w) were $11 \%, 57 \%$ and $114 \%$.

\section{Figure 1.}

After that, the full factorial design using Minitab software with 18 iterations were performed to identify the functional system and find the optimal solutions (Table 1). The functional system included the maximum v.M stress on the plate, maximum v.M stress on the screws, maximum displacement and the weight of the fixation structure (i.e. weight of screws and plate). In Table 
1.A, the values of the design variables in each model are listed. In order to reduce the number of models, the amount of plate length, number of holes and distances between the centers of plate holes were considered fixed.

\section{Table 1.}

The commercial finite element software, Abaqus (Simulia, Dassault Systems) was used for analysis. Isotropic linear elastic materials were assigned for bone, plate and screws. ${ }^{14,24}$ The interaction between all components was considered as "tie". ${ }^{24}$ Poisson's ratio and modulus of elasticity were respectively defined to be 0.3 and $210 \mathrm{GPa}$ for plate/screws and 0.3 and $17 \mathrm{GPa}$ for the bone. ${ }^{26}$ A $0.2 \mathrm{MPa}$ compression pressure was applied to the upper part of the bone, while the distal part of the bone was fixed. ${ }^{24}$ The loading conditions selected in this paper were in accordance with the load on the bones of the lower limbs in real conditions, and this type of loading has been used in many numerical and experimental studies (e.g. ${ }^{24}$ ). Two groups of bones with two different diameters of 20 and $40 \mathrm{~mm}$ were used to investigate the effect of the bone diameter and the amount of applied load on the outputs. The equivalent force applied to the center of pressure was 17.4 $\mathrm{N}$ for the smaller bones and $36.2 \mathrm{~N}$ for the larger ones. Since weight bearing on a broken bone changes over time, the values of dimensionless v.M stress were obtained using two different load values. These load values are corresponding to the initial stages of healing in which a person uses a cane or other aids, as too soon weight bearing may lead to problems. ${ }^{27}$

Quadratic tetrahedral three-dimensional stress type elements (C3D10) were used to mesh the two parts of the bone, and linear hexahedral elements (C3D8R) were utilized for the screws and plates. Finally, a convergence analysis showed an almost independence of the parameters of interest on the mesh size (i.e. less than $5 \%$ for displacement and v.M stress). For plate with thickness of 2, 10 and $20 \mathrm{~mm}$, the element size was selected $0.3,0.4$ and $0.6 \mathrm{~mm}$, respectively, and 
for screws with diameters of 2, 8 and $15 \mathrm{~mm}$, the element size was selected $0.2,0.4$ and $0.5 \mathrm{~mm}$, respectively. Figure 2 shows the CAD and meshed model created in this study.

\section{Figure 2.}

After FE modeling, the non-dominated sorting genetic algorithm (NSGA-II), which is a multiobjective optimization algorithm, was applied to find the optimal solutions. There were two design variables including $\mathrm{d} / \mathrm{w}$ and $\mathrm{t} / \mathrm{w}$, and two objectives (cost functions) which were the weight of the fixation system and the maximum v.M stress on the structure. These cost functions were obtained based on the multivariable regression coefficients from normalized data. Table 2 summarizes the GA cost functions, constraints and optimization parameters obtained from a trial and error and ref. $^{28}$. Also, a population size of 300 was considered by computing several population sizes and comparing the outcomes.

\section{Table 2.}

\section{Results}

\subsection{Model Validation}

Ahmad et al. ${ }^{8}$ investigated the effect of the gap between the plate and bone on the failure load of the fixation structure. The results showed that the amount of failure load decreased when the gap increased. In the current study, Ahmad's model was used as a reference for validation. The effect of distance on the maximum v.M stress was studied using a 3D FE analysis under similar loading conditions as applied in Ahmad's model. The results of FE analysis showed that with increasing the plate distance from the bone, the maximum stress on the structure increased. 
While in Ahmed's model ${ }^{8}$ the amount of failure load was reported, in this study the maximum v.M stress was evaluated. According to Equations 1-3, the maximum amount of stress on the structure is inversely related to the amount of failure load:

$\frac{F_{\text {applied }}}{F_{\text {failure }}}=\frac{\sigma}{\sigma_{\text {ultimate }}}$

$F_{\text {failure }}=\sigma_{\text {ultimate }} \times F_{\text {applied }} \times \frac{1}{\sigma}$

$F_{\text {failure }}=\alpha \times \frac{1}{\sigma}$

where $F_{\text {applied }}$ and $F_{\text {failure }}$ represent the applied force on the bone and the failure load, respectively. $\sigma$ and $\sigma_{\text {ultimate }}$ are the values of the maximum v.M stress and ultimate stress. $\alpha$ is a constant coefficient obtained by equations 1 and 2 . Normalized values of the inverse of maximum v.M stresses and failure loads were used to compare the results. The normalized values of failure load, the inverse of the maximum v.M stress and displacement are obtained by dividing these values by the largest value of failure loads, inverse stresses and distances, respectively. As shown in Fig. 3, the two models were in close agreement. Also, R-squares calculated from a linear regression of the models of this study and ref. ${ }^{8}$ were 0.96 and 0.94 , respectively, suggesting a linear behavior in both models.

\section{Figure 3.}

\subsection{Main and Interaction Effects}

Fig. 4 illustrates the normalized amount of the maximum v.M stresses on the structure and the ratio of the maximum v.M stress on the plate to the screw, for two bones with diameters of 20 and $40 \mathrm{~mm}$. The amount of equivalent force applied on these two bones is 17.4 and $36.2 \mathrm{~N}$, 
respectively. According to these figure, the size of the bone has not a significant effect on the maximum v.M stress and the ratio of maximum stress on plate to screw. Also, the maximum difference between the values obtained from two different sizes of bone was less than $10 \%$. Therefore, the size of the bone and the amount of load on it did not have a significant effect on the results. As a result, only the outputs of the bone with a diameter of $40 \mathrm{~mm}$ have been used in the coming analysis.

\section{Figure 4.}

Figure $5 \mathrm{a}$ illustrates the effect of $\mathrm{t} / \mathrm{w}$ and $\mathrm{d} / \mathrm{w}$ ratios on the plate normalized stress. By increasing $\mathrm{d} / \mathrm{w}$ from $11 \%$ to $46 \%$, the maximum stress on plate decreased by about $8.3 \%$, while by further growth in $\mathrm{d} / \mathrm{w}$ from $46 \%$ to $86 \%$, the stress increased by about $232 \%$. This trend shows that increasing the diameter of screw does not necessarily reduce the stress on the plate.

\section{Figure 5.}

The effect of increasing t/w ratio on both stresses and maximum displacements was similar; i.e. by increasing this ratio from $11 \%$ to $114 \%$, the amounts of normalized v.M stress on the plate, normalized v.M stress on screw, maximum displacement and the maximum v.M stress on structure decreased by $96 \%, 83 \%, 99 \%$ and $92 \%$ respectively (Figs. 5a-d). As the d/w increased from $11 \%$ to $46 \%$, the amounts of the maximum v.M stress on the screws, maximum displacement and the maximum v.M stress on the structure decreased by about $83 \%, 15 \%$ and $56 \%$ respectively. By further growth in $\mathrm{d} / \mathrm{w}$ from $46 \%$ to $86 \%$, the amount of maximum v.M stress on screw declined by about $23 \%$, while the amounts of maximum displacement and maximum v.M stress on the structure increased by about $64 \%$ and $232 \%$, respectively (Figs. 5b, 5c and 5d).

According to Fig. 6, in all t/w levels, with the growth of $d / w$ to a certain level, the amount of stress on the structure reduced, while by further increase in $d / w$, the amount of stress on the plate increased. On the other hand, by increasing the value of $\mathrm{t} / \mathrm{w}$ in all $\mathrm{d} / \mathrm{w}$ ratios, the maximum amount 
of stress on the structure declined. Therefore, it was observed that the maximum v.M stress on the structure depends on the values of $\mathrm{t} / \mathrm{w}$ and $\mathrm{d} / \mathrm{w}$, and a similar behavior can be seen in each t/w; i.e. at any ratio of $\mathrm{t} / \mathrm{w}$, by changing $\mathrm{d} / \mathrm{w}$ from 0.11 to 0.46 , the maximum $\mathrm{v} . \mathrm{M}$ stress on the structure decreased, while by further growth in $\mathrm{d} / \mathrm{w}$ from 0.46 to 0.86 , v.M stress increased.

\section{Figure 6.}

Figure 7 demonstrates the effect of different $t / w$ and $d / w$ values on the maximum v.M stress

on the plate. According to this figure, with increasing the thickness of the plate, the amount of stress on the plate decreased. Also, in general, increasing the diameter of the screw first reduced the stress and further increasing the diameter elevated the stress on the plate. In addition, it is worth to note that a structure with a lower weight did not always have a more v.M stress. For example, the least amount of v.M stress was observed for $\mathrm{t} / \mathrm{w}=114 \%$ and $\mathrm{d} / \mathrm{w}=46 \%$ (see Fig. $7 \mathrm{f}$ ), while the heaviest model is shown in Fig. 7i with a larger maximum stress with respect to that of Fig. 7f. The largest amount of v.M stress was related to the plate with the least thickness and the largest screw (Fig. 7g), while the minimum weight was related to Fig.7a.

\section{Figure 7.}

\subsection{System identification}

In order to identify the fixation structure, the mathematical relation between design variables (t/w and $d / w)$ and three outputs were obtained using a multivariate regression. As mentioned before, the bone diameter had no significant effect on the normalized outputs. Therefore, the analysis was performed using two design variables. Table 3 shows the regression characteristics.

\section{Table 3.}


Based on Table 3, the cost functions 1 (the weight of the structure) and 2 (the maximum v.M stress on the structure) in terms of two variables were obtained (equations 4 and 5).

Weight $=0.5109(x)+0.5568(y)-0.0774$

Max.v.M stress $=0.2649(x)-2.0828(y)-0.0449\left(\frac{x}{y}\right)+0.0324\left(\frac{1}{x}\right)+1.4164\left(y^{2}\right)+$ $0.1572\left(\frac{y^{2}}{x}\right)+0.4170$

where $\mathrm{x}=\mathrm{t} / \mathrm{w}$ and $\mathrm{y}=\mathrm{d} / \mathrm{w}$.

\subsection{Optimization:}

In order to find the optimal solutions in terms of the maximum stress and weight of the fixation sturcture, an optimization process was performed. The aim was to obtain a system which can withstand high stresses with a minimum weight. Multivariable regression formulas, which were obtained from Table 3, were used to define the cost functions. The NSGA-II results converged after 105 generations and the outcome of analysis is plotted in Fig. 8. Figure 8a illustrates all the optimal and non-optimal solutions. The optimal or non-dominated solutions are the solutions that provide a suitable compromise between all cost functions, and these solutions are not dominated by any other solutions. These non-dominated solutions represent the answers that the structure can withstand a certain amount of stress with the lowest possible weight. Figures $8 b-\mathrm{d}$ demonstrate the stress contours on the fixation structure and values of $\mathrm{t} / \mathrm{w}, \mathrm{d} / \mathrm{w}$, normalized weight and normalized 
stress for 3 different points. According to these figures, as the amount of weight increased by about $64 \%$, the maximum amount of stress decreased by more than $50 \%$.

\section{Figure 8.}

In Table 4, the values for normalized weight, normalized maximum stress, $\mathrm{t} / \mathrm{w}$ and $\mathrm{d} / \mathrm{w}$ for three different ranges of optimal answers are given. According to this table, the range 1 is related to solutions which the normalized weight of the fixation system was under $10 \%$ while the maximum normalized stresses are between $31 \%$ and $50 \%$. The solutions in range 2 demonstrate the points with more normalized weight amount and less maximum normalized stress than those of range 1 . Third range demonstrate the points with the minimum amount of the maximum v.M stress on the structure. In this range, the amount of normalized weight was between $52 \%$ and $71 \%$, while the maximum normalized stresses were less than $1 \%$.

\section{Table 5.}

Figure 9 shows the normalized values of the difference between the maximum v.M stresses on the plate and on the screws, and the normalized maximum v.M stresses on the structure in different weights. According to this figure, the lower the maximum stress difference on the plate and screw, the lower the maximum stress on the structure. This is because when the plate and screws are made by the same material, it would be more efficient to have a relatively equal amount of stress on the screws and plate since the yield strengths for these two parts are equal. In other words, at a certain weight, approaching only one of the fixation parts to its yield point would not be optimal. As a result, according to this diagram, point $\mathrm{C}$ had the lowest maximum stress value, and also, the difference between the stress on the plate and on the screws was less than that for the other points. 
The normalized weight and the maximum normalized stress of this point were equal to $72 \%$ and $0.6 \%$, respectively. Also, $\mathrm{t} / \mathrm{w}$ and $\mathrm{d} / \mathrm{w}$ ratios were $114 \%$ and $46 \%$, respectively. Point $\mathrm{B}$ was the second minimum point in terms of the maximum stress and the difference between the stress on the plate and on the screw. While the weight of the point $\mathrm{B}$ was less than that of point $\mathrm{C}$, the maximum stress of Point B was more than the maximum stress in Point C. Point B had $41 \%$ of the maximum weight of the structure and $1.8 \%$ of the maximum v.M stress with the $t / w=57 \%$ and $\mathrm{d} / \mathrm{w}=46 \%$.

\section{Figure 9.}

\section{Discussion}

\subsection{Analysis of results}

The results of the simulations performed in this study had a good agreement with results of ref. [6]. According to Fig. 3, with increasing the normalized distance from the lowest value of zero to the maximum value of 1 , the amount of failure load in the Ahmad's model decreased by about $64 \%$ and in this study, it decreased by about $52 \%$. Furthermore, the obtained linear regression equations had $\mathrm{R}^{2}$ values of more than $94 \%$, indicating that both Ahmed's model ${ }^{8}$ and the model developed in this study had a similar linear trend. The model of this study used some simplified assumptions such as the tie constraint between the components (i.e. plate-screw and bone-screw), which caused small differences compared to the results of ref. ${ }^{8}$.

The main effects diagram as shown in Fig. 5a explains that with the growth in $\mathrm{t} / \mathrm{w}$ ratio, the maximum stress on the plate declined. This is due to increase of the cross-sectional area of the plate. However, it is important to note that due to cost and medical restrictions, increasing plate thickness is not always possible. 
In Figs. 5a-c and 6, it was illustrated that by increasing the value of d/w by more than $45 \%$, the maximum v.M stress on the plate, maximum displacement and maximum stress on the structure increased due to the concentration of stress on the edge of the plate. Hence, it is recommended to design the plates and screws such that this ratio becomes about $50 \%$.

Figure $5 b$ illustrates the effect of $\mathrm{d} / \mathrm{w}$ and $\mathrm{t} / \mathrm{w}$ values on the maximum v.M stress of the screws. By increasing the screw diameter, its cross-sectional area increased and the amount of stress on it reduced. With the growth in $\mathrm{t} / \mathrm{w}$, the maximum stress on the screws decreased. This is because the contact area between the screws and the plate increased, and as a result, the maximum amount of stress on the screw decreased. In other words, as the plate thickness reduced, the force was applied to a smaller contact area between the plate and screws, and hence the stress on the screws increased.

As previously mentioned, the bone diameter had no significant effect on the normalized outputs. Also, it did not have any effect on the weight of the fixation structure. Therefore, the problem was formulated without the bone diameter, and two other variables including $\mathrm{t} / \mathrm{w}$ and $\mathrm{d} / \mathrm{w}$ were considered. Figure 8 shows that there was no a unique optimal solution in terms of the weight of the fixation system and the maximum stress. The reason for this is that the minimum weight and the maximum stress on the fixation structure were in inverse relationship with each other. In other words, it was not possible to find one point that had both the lowest weight and the lowest amount of stress. Also, according to this diagram, the amount of the maximum stress did not always decrease by increasing the weight of fixation system. For example, at a constant t/w, by increasing the diameter of screws, the weight of the system increased but due to stress concentration on the plate, the maximum v.M stress increased too.

Since the fracture of the stabilization system would cause many problems for the patient, the first goal is to design a structure that greatly reduces the failure risks; i.e. a fixation system which can withstand higher forces would be desirable. Therefore, according to Fig. 8, ranges 2 and 3 are 
considered optimal points in which the maximum normalized stress on the structure was less than $4 \%$. The values of $\mathrm{d} / \mathrm{w}$ for the optimal ranges were between $37 \%$ and $59 \%$ and the values of $\mathrm{t} / \mathrm{w}$ were more than about $30 \%$. For the cases where minimizing the weight is the main objective, the solutions of range 1 with normalized weight of less than $10 \%$ are proposed.

\subsection{Limitations}

In this work, the width and the length of the plate were assumed constant for all the models to reduce the number of simulations and computational costs. Furthermore, in order to simplify the model, only compressive loading was used to carry out the computer modelling. Although compression load is the most important and greatest force on the bones, the human body are under various loading conditions such as tension, compression, torsion, and shear. Also, the passive/active forces of muscles and tissues around the bone were neglected and the bone was modeled with a hollow cylinder.

\section{Conclusion}

A plate-screw fixation structure for the purpose of bone fracture was analyzed. To come with an optimal design, three design variables including the ratio of the screw diameter to the plate width $(\mathrm{d} / \mathrm{w})$, the ratio of the plate thickness to the plate width (t/w) and the size of the bone were the selected parameters for analysis. Results showed that by increasing the value of $\mathrm{t} / \mathrm{w}$, the maximum v.M stress on the structure always decreased, while with the growth in the $\mathrm{d} / \mathrm{w}$ ratio more than a specific amount, the maximum stress on the structure increased. Furthermore, the diameter of the bone did not have a significant effect on the normalized stresses. In other words, the optimal solutions found in this study were independent of the bone diameter and the amount of force applied on it. Finally, the optimal solutions in terms of the weight and the maximum amount of stress were obtained from an optimization process by a NSGA- II. 


\section{Reference}

1. Idkaidek, A. \& Jasiuk, I. Cortical bone fracture analysis using XFEM - case study. Int. $j$. numer. method. biomed. eng. 2017; 33: e2809.

2. Zimmermann, E. A., Gludovatz, B., Schaible, E., Busse, B. \& Ritchie, R. O. Fracture resistance of human cortical bone across multiple length-scales at physiological strain rates. Biomaterials. 2014; 35: 5472-5481.

3. Zioupos, P., Currey, J. D. \& Casinos, A. Tensile fatigue in bone: Are cycles-, or time to failure, or both, important? J. Theor. Biol. 2001; 210: 389-399.

4. Ehlinger, M., Ducrot, G., Adam, P. \& Bonnomet, F. Distal femur fractures . Surgical techniques and a review of the literature. Orthop. Traumatol. Surg. Res. 2013; 99: 353-360.

5. Claes, L. Biomechanical Principles and Mechanobiologic Aspects of Flexible and Locked Plating. J. Orthop. Trauma. 2011; 25: 4-7.

6. Perren, S. Evolution of the internal fixation of long bone fractures - The scientific basis of biological internal fixation: Choosing a new balance between stability and biology. The Bone \& Joint Journal. 2002; 84: 1093-1110.

7. Duda, G. N., Hoffmann, J. E. \& Kassi, J. Interfragmentary movements in the early phase of healing in distraction and correction osteotomies stabilized with ring fixators. Langenbecks Arch. Surg. 2003; 387: 433-440.

8. Ahmad, M., Nanda, R., Bajwa, A. S. \& Candal-couto, J. Biomechanical testing of the locking compression plate : When does the distance between bone and implant significantly reduce construct stability ?. Injury. 2007; 38: 358-364.

9. Henderson, C. E., Kuhl, L. L., Fitzpatrick, D. C. \& Marsh, J. L. Locking Plates for Distal Femur Fractures : Is There a Problem With Fracture Healing ?. J. Orthop. Trauma. 2011; 
25: $8-14$.

10. Bigdeli, A. \& Damghani Nouri, M. Experimental and numerical analysis and multiobjective optimization of quasi-static compressive test on thin-walled cylindrical with internal networking. Mech. Adv. Mater. Struct. 2019; 26: 1644-1660.

11. Bigdeli, A. \& Nouri, M. D. A crushing analysis and multi-objective optimization of thinwalled five-cell structures. Thin-Walled Struct. 2019; 137: 1-18.

12. Lee, C., Shih, K., Hsu, C. \& Cho, T. Simulation-based particle swarm optimization and mechanical validation of screw position and number for the fixation stability of a femoral locking compression plate. Med. Eng. Phys. 2013; 36: 57-67.

13. Moazen, M. et al. Rigid versus flexible plate fixation for periprosthetic femoral fractureComputer modelling of a clinical case. Med. Eng. Phys. 2012; 34: 1041-1048.

14. Wee, H., Reid, J. S., Chinchilli, V. M. \& Lewis, G. S. Finite Element-Derived Surrogate Models of Locked Plate Fracture Fixation Biomechanics. Ann. Biomed. Eng. 2017; 45: 668-680.

15. Taghizadeh, E. et al. Biomechanical Role of Bone Anisotropy Estimated on Clinical CT Scans by Image Registration. Ann. Biomed. Eng. 2016; 44: 2505-2517.

16. Taghipoor, H. \& Damghani Nouri, M. Experimental and numerical investigation of lattice core sandwich beams under low-velocity bending impact. J. Sandw. Struct. Mater. 2019; 21: $2154-2177$.

17. Taghipoor, H. et al. Experimental and numerical study of lattice-core sandwich panels under low-speed impact. in Materials Today: Proceedings 2020; 27: 1487-1492.

18. Taghipoor, H. \& Noori, M. D. Experimental and numerical study on energy absorption of lattice-core sandwich beam. Steel Compos. Struct. 2018; 27: 135-147.

19. Eyvazian, A. et al. Experimental and numerical investigations on axial crushing of square cross-sections tube with vertical wave. Steel Compos. Struct. 2020; 36: 119-141. 
20. Taghizadeh, E., Terrier, A., Becce, F., Farron, A. \& Büchler, P. Automated CT bonesegmentation using statistical shape modelling and local template matching. Comput. Methods Biomech. Biomed. Engin. 2019; 22: 1303-1310.

21. Dao, S. D., Abhary, K. \& Marian, R. A bibliometric analysis of Genetic Algorithms throughout the history. Comput. Ind. Eng. 2017; 110: 395-403.

22. Ferguson, S. J., Wyss, U. P. \& Pichora, D. R. Finite element stress analysis of a hybrid fracture fixation plate. Med. Eng. Phys. 1996; 18: 241-250.

23. Stoffel, K., Dieter, U., Stachowiak, G., Gächter, A. \& Kuster, M. S. Biomechanical testing of the LCP - how can stability in locked internal fixators be controlled ?. Injury. 2003; 34: 11-19.

24. Mohandes, Y., Tahani, M., Rouhi, G. \& Tahami, M. A mechanobiological approach to find the optimal thickness for the locking compression plate: Finite element investigations. Proc. Inst. Mech. Eng. Part H J. Eng. Med. 2021.

25. Nassiri, M., Macdonald, B. \& Byrne, J. M. O. Computational modelling of long bone fractures fixed with locking plates e How can the risk of implant failure be reduced ? J. Orthop. 2013; 10: 29-37.

26. Wang, C. J., Yettram, A. L., Yao, M. S. \& Procter, P. Finite element analysis of a Gamma nail within a fractured femur. Med. Eng. Phys. 1998; 20: 677-683.

27. Kubiak, E. N., Beebe, M. J., North, K., Hitchcock, R. \& Potter, M. Q. Early weight bearing after lower extremity fractures in adults. Journal of the American Academy of Orthopaedic Surgeons. 2012; 21: 727-738.

28. Ehsani, A. \& Rezaeepazhand, J. Stacking sequence optimization of laminated composite grid plates for maximum buckling load using genetic algorithm. Int. J. Mech. Sci. 2016; 119: 97-106. 


\section{Appendix}

The Table 1A shows the model number and the value of design variables for each model. The models that have similar $\mathrm{t} / \mathrm{w}$ and $\mathrm{d} / \mathrm{w}$ values, have the same model number. It should be mentioned that the models number in the Fig. 4 are based on this table.

\section{Table 1A.}




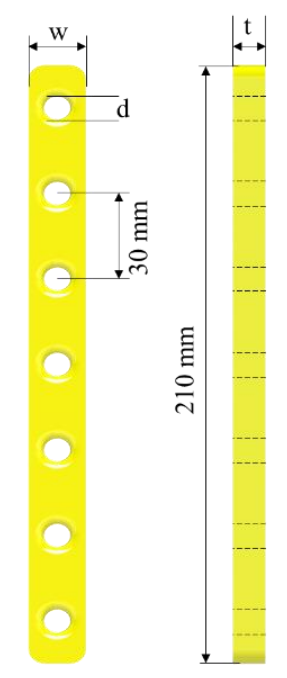

Figure 1. Two of design variables ( $t$ and $d$ ) and the plate dimensions.

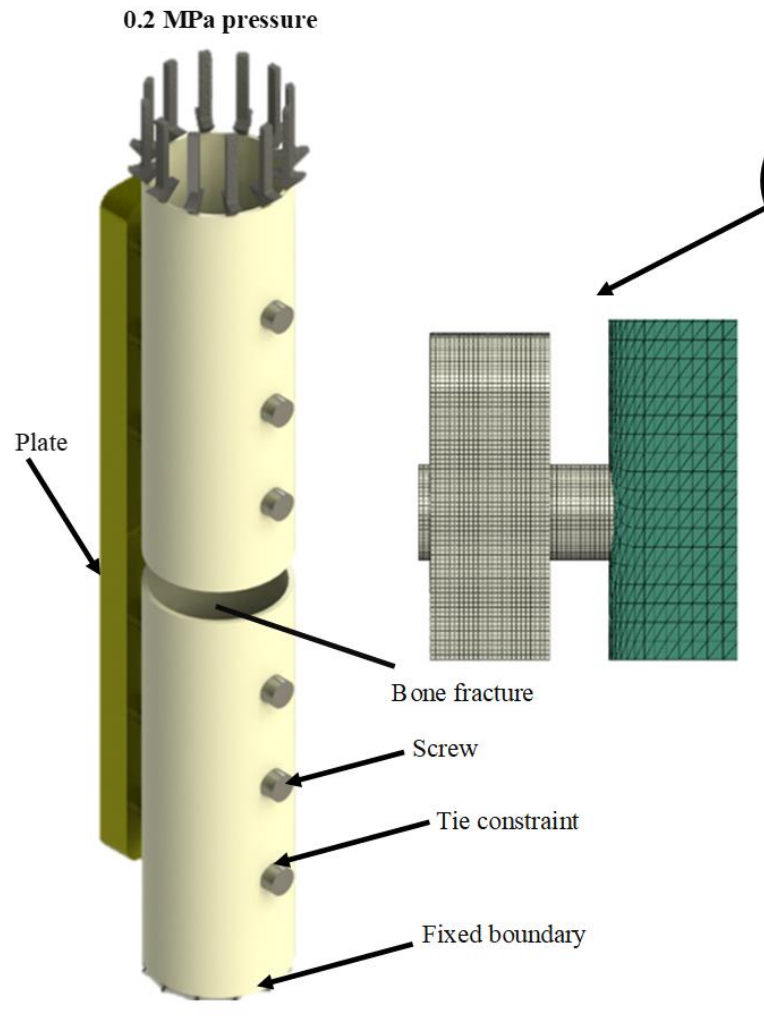

(a)

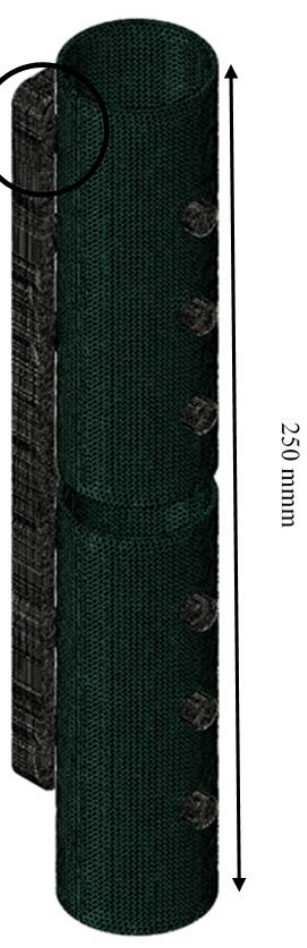

(b)

Figure 2. Fixation structure: (a) the CAD model and (b) the meshed model. 


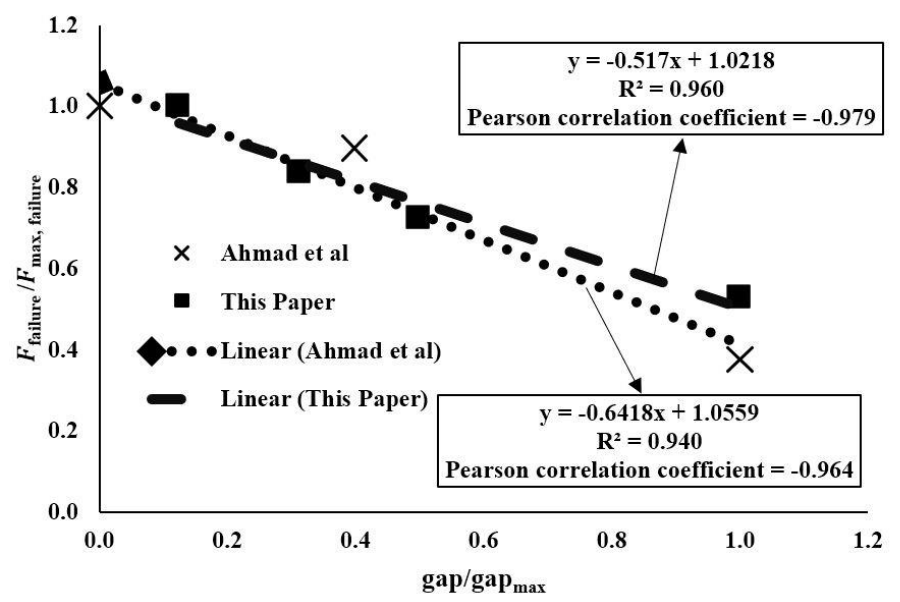

Figure 3. Comparison of failure loads obtained from FE of the current study with that of Ahmad et al.8 as a function of dimensionless gap between the plate and bone.

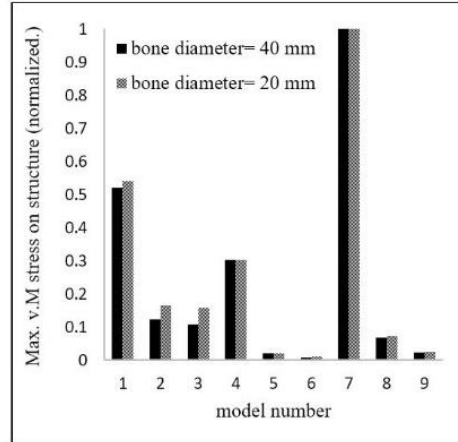

(a)

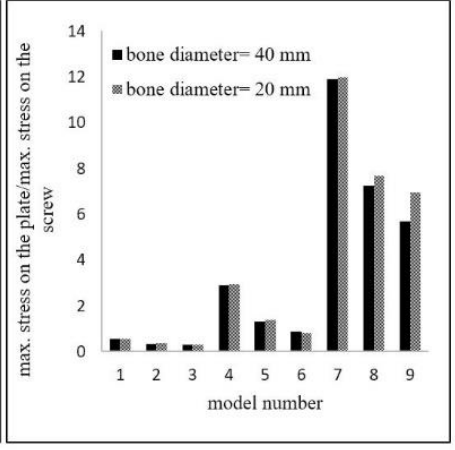

(b)

Figure 4. The effect of bone size on the (a) maximum normalized v.M stress on the structure and (b) the ratio of maximum v.M stress on plate to maximum v.M stress on screws. The maximum difference between the results for two bone diameters was about $10 \%$.
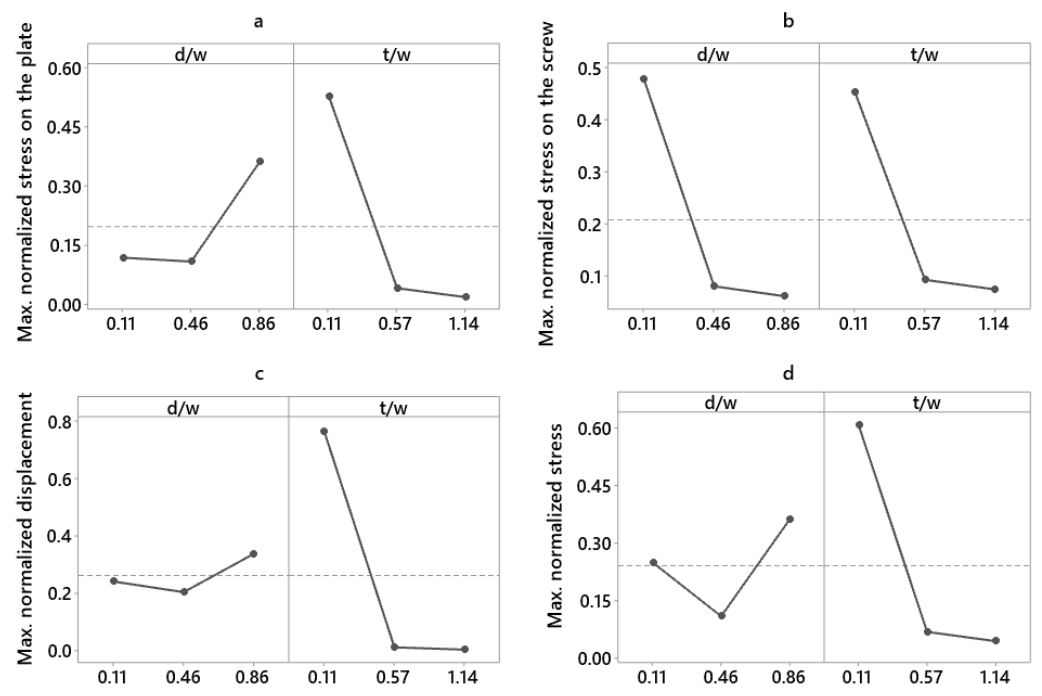
Figure 5. Main effects plots with mean normalized data for: (a) maximum plate stress, (b) maximum screw stress, (c) maximum displacement, and (d) maximum stress on the structure.

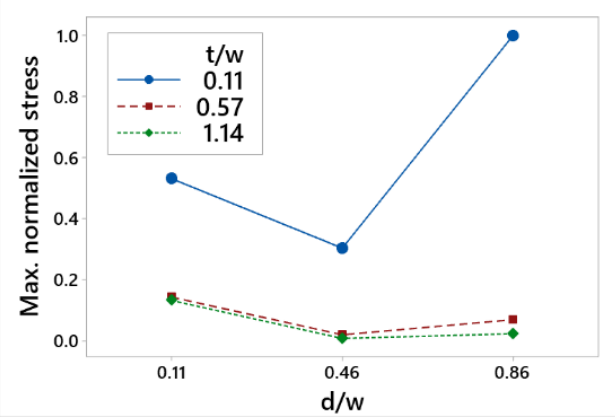

Figure 6. Interaction plot for the maximum v.M stress on the structure.

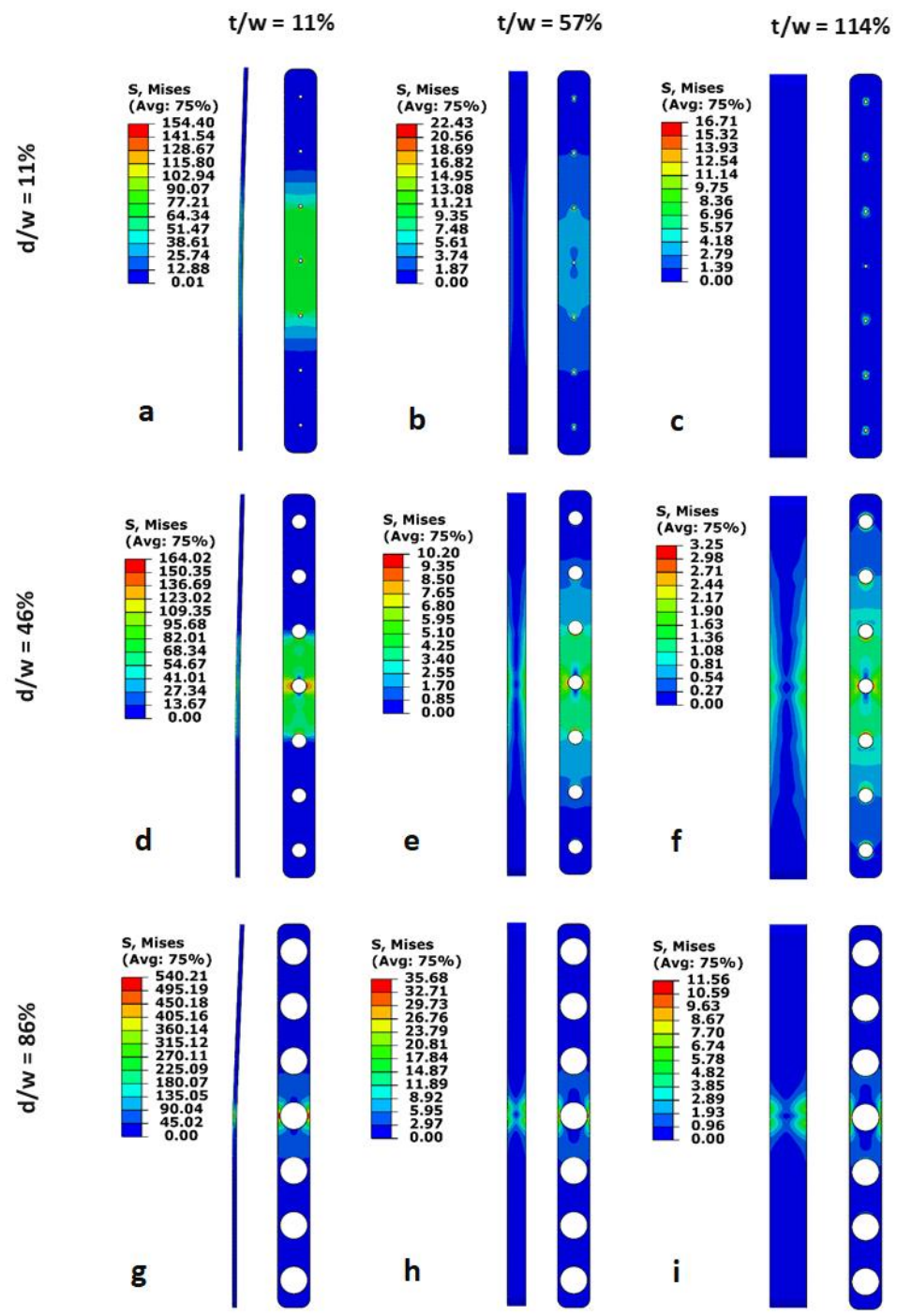

Figure 7. The v.M stress contours on the plate in different $t / w$ and $d / w$ ratios. $f$ had the lowest and $g$ had the highest v.M stress on the plate. The lightest and heaviest models were $a$ and $i$, respectively. The scale of deformations is $1: 1$ and the stress values are in MPa. 


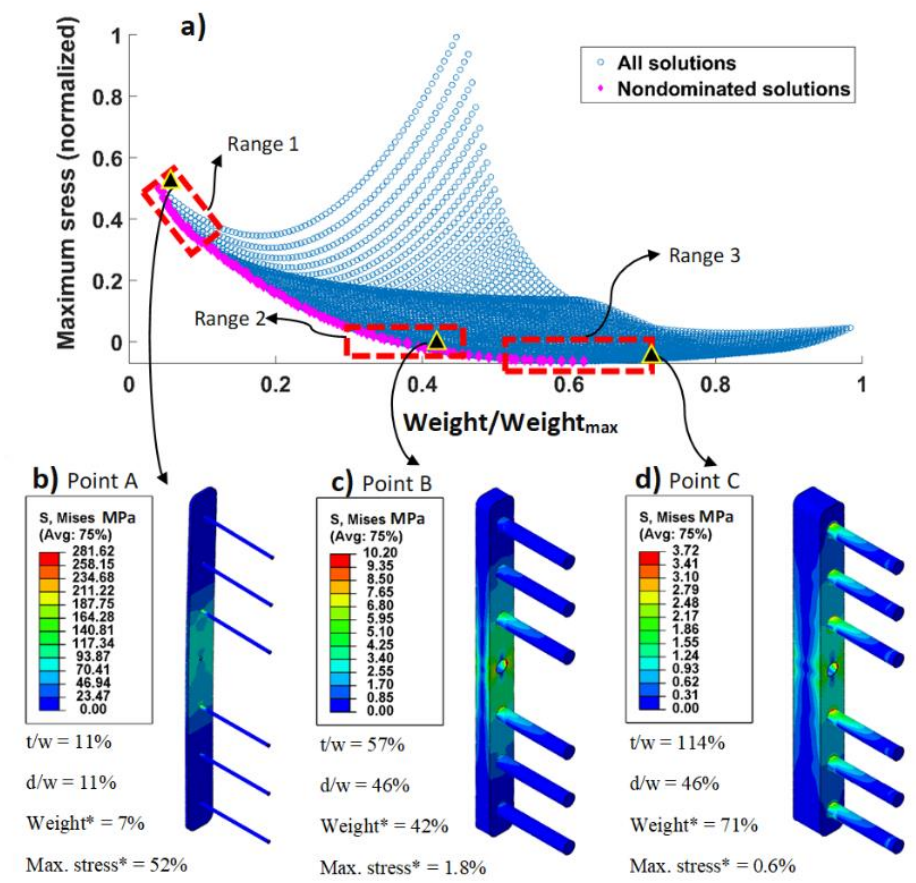

Figure 8. Resultant Pareto Front of the optimization and stress contours for three representative configurations: (a) All solutions and Pareto Front, (b) point A, (c) point B, and (d) point C. Points A-C are sample solutions from all solutions. *Normalized values.

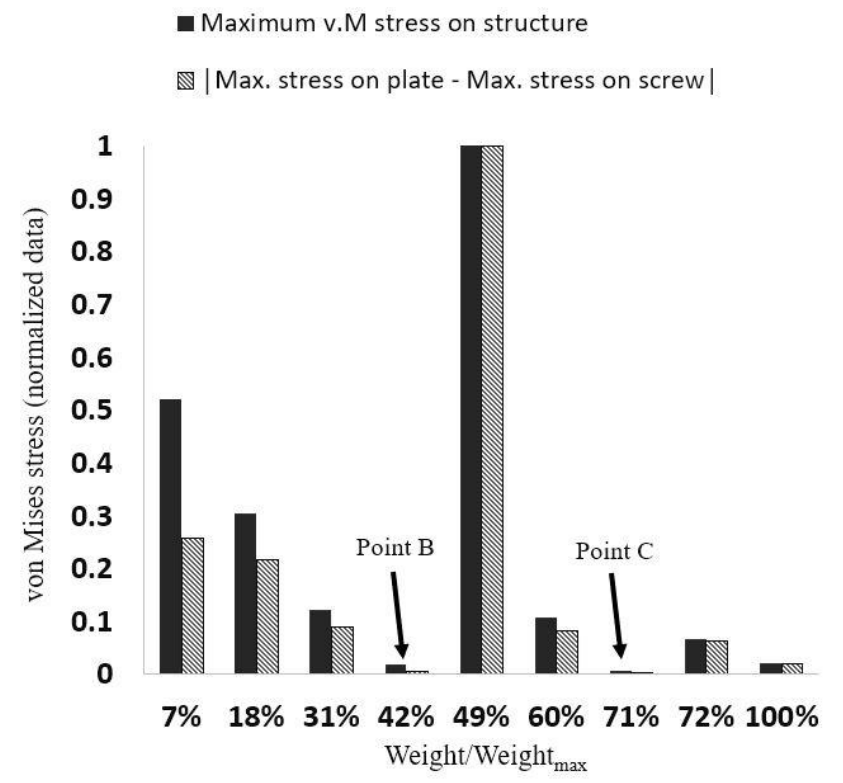

Figure 9. Normalized values of the difference between the maximum v.M stress on the plate and on the screws, and the normalized maximum v.M stresses on the structure in different weights. Point $B$ and point $C$ are the points in which the minimum amount of normalized maximum v.M stress on the fixation system was less than $2 \%$. 